\title{
A Computational Investigation of the Properties of a Reverse Osmosis Membrane
}

\author{
Zak E. Hughes and Julian D. Gale
}

May 19, 2010

Nanochemistry Research Institute, Department of Chemistry, Curtin

University of Technology, P.O. Box U1987, Perth, WA 6845, Australia

\begin{abstract}
Reverse osmosis (RO) is currently one of the most widely used methods of desalination in the world and rapidly increasing in usage. The membranes used in the $\mathrm{RO}$ process play a vital role in determining the effectiveness of the desalination process. In this work, fully atomistic molecular dynamics simulations of one of the most widely employed membranes, namely the FT30 polyamide material, have been carried out in order gain greater understanding of the structure of the system and it's interaction with saline solution. The system studied consisted of a solvated membrane layer and a layer of bulk solution, thus allowing the membrane interface to be simulated. The behaviour of water and salt ions in both the bulk solution and membrane has been investigated. It was found that the diffusivities of water and the salt ions were reduced by an order of magnitude within the membrane. Furthermore, umbrella sampling methods have been used in order to determine the free energy surface associated with the salt ions passing through the membrane-solution interface. The present work demonstrates that there is a high degree of variability in the barrier to salt diffusion into the membrane associated with the structure of the water as it permeates the membrane. Despite this variability in the free energy gradient, all cases ultimately exhibit a high resistance to ionic diffusion due to charge separation. Migration of a sodium cation/chloride anion pair fails to substantially lower the barrier to salt diffusion, thus confirming the robust nature of the membrane selectivity for water.
\end{abstract}

\section{Introduction}

Desalination of seawater and brackish water is an area that is growing in importance. The increasing population of the world and changes to the climate 
and environment mean that traditional water sources are being placed under increasing pressure[1, 2, 3, 4]. Desalination of brackish water and seawater is one method of tackling this problem, albeit at the cost of increased energy usage.

Early desalination methods were based on thermal processes, and in some parts of the world these methods are still extensively used[2]. However, the high energy costs associated with such processes has led to a number of different desalination techniques being developed[1, 2]. The most popular current technique is that of reverse osmosis $(\mathrm{RO})$, where saltwater is forced through an appropriate membrane at high pressure, allowing the water to pass through, while the salt ions are largely rejected. Typically, a single pass through the current membranes can achieve approximately two orders of magnitude in reduction of the salt concentration[1,5]. Obviously the membrane material plays a highly significant role in determining the success of the RO process. Understanding how the different species in the feedwater interact with the membrane will allow new, more efficient membranes to ultimately be designed.

The first widely used commercial RO membranes were made of celluloseacetate (CA), but these membranes have a number of drawbacks[6]. Firstly, they tend to compact under high pressure reducing the performance of the membrane. The second even more serious problem is that cellulose-acetate membranes deteriorate by hydrolysis; this is a particular problem at high or low $\mathrm{pH}$. Thus to ensure a good lifetime for a CA membrane it was necessary to monitor and control the $\mathrm{pH}$ of the feedwater. Because of these disadvantages, CA membranes have largely been replaced by a new generation of composite polyamide-polysulfone (PA-PS) membranes. In these membranes the active polyamide layer, with a thickness of the order of $2000 \AA[7,8,9]$, is responsible for the actually removal of salt from the seawater while the much thicker $(\sim 50 \mu \mathrm{m})$ polysulfone layer simply provides structural support and strength, see Figure 1. Despite the fact that there has been a large amount of work done studying composite membranes, there are still many aspects of their behaviour that are not fully understood, particularly at the atomistic level. Molecular simulation of reverse osmosis membranes can, therefore, provide important information about these membranes and how they interact with species in the feedwater that cannot be determined easily by experimental methods. In particular, simulation can provide important information about how membranes may be altered in order to have a greater flow rate or be made more resistant to foulants present in the feedwater. However, before such modifications can be proposed the standard behaviour of these membranes needs to be determined.

Most previous molecular simulations of reverse osmosis have abstracted the membrane structure, coarse-graining it in some way[10, 11, 12, 13]. There have only been a few studies where the membrane has been simulated in full atomistic detail[5, 14]. The work of Kotelyanskii et al. [5] provided valuable information about how water diffuses within one particular RO membrane, but due to the computational resources available at that time was limited to simulating a relatively small system of solvated polyamide (62 repeat units). In addition, this work only simulated the bulk membrane, such that no information about the behaviour at the membrane interface was provided. The systems generated by 
Harder et al.[14] started with monomers which were polymerised during the course of the simulation. This method did generate configurations where an there was an interface between the bulk solution and the membrane, but how salt ions might interact with the membrane was not investigated.

In both these previous simulations the polyamide modelled was FT30[15, 16], for the simple reason that this material is experimentally the best characterised of the polyamide membranes. Even so, many details of the structure of FT30 are still poorly understood. FT30 consists of two different monomers, trimesoylchloride (TMC) and $m$-phenylenediamine (MPD), that are cross-linked to each other; Figure 2 shows the structure of a single repeat unit.

The motivation for the present study is to investigate, for the first time, the behaviour of species in bulk solution, in the membrane, and at/across the membrane interface at the atomistic level. Thus the systems simulated need to consist of both a membrane layer and a solution layer. A further aim was to attempt to model the polysulfone part of the membrane in some manner, which has previously been neglected in atomistic models. With these goals in mind, a series of simulations of the FT30 polymer have been performed. The arrangement of the system and the salt concentration present in both the bulk solution or/and with the membrane itself have been varied in order to observe differences in the behaviour of the system. These simulations provide important information about the diffusion rates and coordination of species in both bulk solution and within the membrane. This fundamental knowledge aims to provide the insights necessary to lead ultimately to improved membrane design for greater energy efficiency.

\section{Methods}

Due to the complex nature and large size of the system of interest, and the fact that many of the properties of relevance are dynamic, the most appropriate computational method for investigating the membrane is to use classical molecular dynamics simulations (MD). While it would be possible to use a coarse-grained model for the membrane, which would allow greater length- and time-scales to be simulated, this was rejected for two reasons. Firstly, it is at the atomistic level that information on the membrane structure and behaviour is hardest to determine from experiment. Secondly, constructing a coarse-grained model that accurately describes FT30 is extremely difficult based on the current information; indeed any such coarse-graining process would typically need data determined from atomistic simulations.

The polyamide membrane was modelled using the well known all atom optimised potentials for liquid simulations (OPLS-AA) force field[17, 18, 19], which has been used to study a range of systems where organic molecules are solvated by water[20, 21, 22, 23, 24, 25]. In this force-field, intramolecular interactions are described by harmonic bond stretching and angle bending terms, the torsional angles by a cosine term, while the intermolecular interactions are represented by a Coulomb interaction and Lennard-Jones potentials. 
For the water molecules, the TIP4P-Ew model[26] was used. This is a rigid four site model consisting of a Lennard-Jones site representing the oxygen atom and three point charges representing the two hydrogens and a Drude particle. This water model describes the most properties of water relevant to this study (i.e. diffusion coefficient, radial distribution function, etc) well at a level of computational expense that is affordable. In addition, Joung and Cheatham[27, 28] recently developed parameters for salt ions specifically for use with TIP4PEw water.

As mentioned previously, it is desirable that the porous polysulfone (PS) scaffold be represented in the simulations in some manner. However, if a fully atomistic representation was used the simulations would increase significantly in computational expense. Instead it was decided that the PS support would be abstracted and represented by a base constructed from a layer of atoms placed on a hexagonal lattice. The atoms in this layer had no partial charge and the parameters for the van der Waal's interactions were set to those of an aliphatic carbon in the OPLS-AA force field (i.e. $\sigma=3.5 \AA, \varepsilon=2.76 \mathrm{~kJ}$ $\left.\mathrm{mol}^{-1}\right)$. These atoms were tethered to their positions by a harmonic potential with a bond constant of $419 \mathrm{~kJ} \mathrm{~mol}^{-1}$. These parameters were chosen so that the support/pore would not interact with the water strongly, ensuring that the diffusion of the water within the system is limited by the polyamide-water interaction.

The MD code used for the simulations was DLPOLY version 2.18[29], with the Velocity Verlet algorithm used to integrate the equations of motion. The Coulomb interactions were evaluated using a smooth particle mesh Ewald sum[30] (with a relative accuracy of $1.0 \times 10^{-6}$ ) and a timestep of $0.5 \mathrm{fs}$ was used. The simulations were carried out in the either the canonical or isothermal-isobaric ensembles using the Nosé-Hoover thermostat and Hoover barostat[31, 32], with relaxation parameters of 1.0 and $2.0 \mathrm{ps}$, respectively.

The unbiased diffusion of salt ions across the membrane interface is not observed on the time scales currently accessible to classical molecular dynamics, as would be expected for the salt rejecting membrane material simulated. In order to gain information about the resistance to salt diffusion through FT30, umbrella sampling methods were used to calculate the free energy barrier for an ion to enter the membrane. These simulations were also carried out using DLPOLY, but modified to incorporate the PLUMED[33] plug-in.

Experimental structural information for FT30 indicates that $~ 19.4 \%$ of the TMC groups are cross-linked and that the water content of the solvated polymer is about $23 \%$ of the system mass [5, 9]. Based on these estimates, a model for the solvated FT30 membrane was constructed. Initially, 24 polymers, each consisting of 23 repeat units, were solvated in water and the system was equilibrated in an $N p T$ ensemble at $300 \mathrm{~K}$ and $1 \mathrm{~atm}$ for $2 \mathrm{~ns}$. Once equilibrated, 54 pairs of carboxylic acid groups that were 5-9 $\AA$ apart were randomly chosen, and connected by an MPD monomer. A further equilibration run of 1 ns under the same conditions was then carried out for the cross-linked material.

Once the solvated membrane was equilibrated, three different arrangements of system were constructed. The first model has the polyamide membrane in a 
2-D layer arrangement, in contact with solution at both surfaces. In the second system, the membrane was placed in contact with the atoms representing the polysulfone support. In the third arrangement, the support was again present, but this time a pore is present in the support. This pore is cylindrical with a radius of $20 \AA$ and the overall pore/support being $12.1 \AA$ deep. Below the pore was another box of water representing the pure filtrate. Figure 3 shows these three different system arrangements. By constructing these three different representations of the RO system, the influence of the support and it's porosity can be examined.

In addition to the three different system arrangements, the membrane was simulated with three distinct "feedwater" conditions; a solution without any salt ions (i.e. pure water), a solution of seawater concentration $(0.55$ molal $\mathrm{NaCl})$ and a dilute salt solution (0.0055 molal), which approximates the feedwater in the second pass through the RO process. A solution at each concentration was equilibrated for $1 \mathrm{~ns}$, the solvated polyamide was placed into this simulation and molecules overlapping with the membrane structure were deleted. A water molecule was considered overlapping if the oxygen is within $2.6 \AA$ of a nonhydrogen membrane atom. The combined membrane and solution were then allowed to equilibrate for a further 1 ns before production runs were started.

The final simulation cells possessed orthorhombic boundary conditions,; $49 \times$ $48.50 \AA$ along the $\mathrm{x}$ and $\mathrm{y}$-axes, respectively, with the length in the $\mathrm{z}$-direction, which represents the direction perpendicular to the plane of the membranesolution interface, varying depending upon the system being simulated. In the case of the systems with either no support or a non-porous support the z-axis was $203 \AA$ with the depth of the membrane layer and the solution layer being approximately equal. For the systems with a porous base the length in the $\mathrm{z}$ direction was $300 \AA$, with the part of the system above the pore having the same initial configuration as the system with the non-porous support.

In order to determine what influence the membrane has, a control run for the solution alone at each of the three concentrations was also performed. The details of all the different simulations performed are summarised in Table 1.

\section{Results and Discussion}

Water and saline solutions In order to have a set of reference data that the FT30 simulations can be compared against, control runs of pure TIP4P-Ew and $\mathrm{NaCl} / \mathrm{TIP} 4 \mathrm{P}-\mathrm{Ew}$ water solutions at two different concentrations, $0.0055 \&$ 0.55 molal, were carried out. The diffusion coefficients of the three species at the three different solution concentrations are given in Table 2. Given the large number of different force fields and water models previously used for simulation of $\mathrm{Na}^{+} / \mathrm{Cl}^{-}$ions in water, it is not surprising that the properties, such as the diffusion coefficient and coordination number, can vary significantly between different works[34].

The experimental value for the self-diffusion of water is $2.3 \times 10^{-9} \mathrm{~m}^{2} \mathrm{~s}^{-1}$, while the diffusion coefficient of water in a simulation will depend not only on the 
model chosen but also the simulation parameters[35]. The diffusion coefficient of a system of pure TIP4P-Ew water, under the simulation conditions used in this paper, was determined to be $2.70 \times 10^{-9} \mathrm{~m}^{2} \mathrm{~s}^{-1}$. This is a slight overestimation of the experimental value, but compares relatively well with other water models. The TIP3P model, used by Harder et al.[14], gives the self diffusion coefficient of water as $5.19 \times 10^{-9} \mathrm{~m}^{2} \mathrm{~s}^{-1}[36]$, while for the CVFF model used in the simulations of Kotelyanskii et al. $[5,37]$ the diffusion rate varies between $2.45-8.02 \times 10^{-9}$ $\mathrm{m}^{2} \mathrm{~s}^{-1}[35]$ depending on the type and distance of cutoff used.

Regarding the salt ions, the experimental values of the diffusion rates at infinite dilution are 1.33 and $2.03 \times 10^{-9} \mathrm{~m}^{2} \mathrm{~s}^{-1}$ for $\mathrm{Na}^{+}$and $\mathrm{Cl}^{-}$, respectively[38], and these values compare well with the diffusion coefficients of the salt ions in the 0.0055 molal solution. Experimental work by Woolf[39] determined the diffusion rates of water, the sodium ion and the chloride anion to be 2.27, 1.28 and $1.85 \times 10^{-9} \mathrm{~m}^{2} \mathrm{~s}^{-1}$, respectively, in a $0.5 \mathrm{M}$ solution of $\mathrm{NaCl}$. Simulation data for the diffusion coefficients at 0.55 molal are more difficult to find as many simulations use salt solutions of higher concentration. One simulation[40] carried out at this concentration, using a flexible SPC water model[41] together with the Smith-Dang[42, 43] salt parameters, determined diffusion coefficients of $2.01,0.88$ and $1.27 \times 10^{-9} \mathrm{~m}^{2} \mathrm{~s}^{-1}$ for water, $\mathrm{Na}^{+}$and $\mathrm{Cl}^{-}$, respectively. Joung and Cheatham[28], using the same water model and salt parameters employed in this paper, calculated $D_{\mathrm{Na}}=1.12 \times 10^{-9} \mathrm{~m}^{2} \mathrm{~s}^{-1}$ and $D_{\mathrm{Cl}}=1.52 \times 10^{-9} \mathrm{~m}^{2} \mathrm{~s}^{-1}$ for a solution of 1 molal concentration. These values accord well with the trend in diffusion coefficients calculated in this study. It is known[44, 40, 45] that the diffusivity of species in a solution decreases as the concentration of the solution increases, and this qualitative behaviour is correctly reproduced. Overall, the model reproduces the qualitative behaviour of salt solutions correctly and is quantitatively of reasonable accuracy for ion diffusion rates.

Aside from the diffusion coefficients, the coordination number of the salt ions within water can differ appreciably between studies. For sodium, values between 4 and 6 have been reported from simulation[40, 46, 34], while a range between 4 and 8 has been seen experimentally. The situation with the chloride ion is similar, simulations placing the coordination number in the range of 7 to 12 waters[34]. Lyubartsev and Laaksonen[40] reported the coordination numbers of a $0.55 \mathrm{M}$ solution (flexible SPC water, Smith-Dang salt ions) being 5.8 and 6.9 for $\mathrm{Na}^{+}$and $\mathrm{Cl}^{-}$, respectively. They also investigated the effect of concentration on the coordination number, finding that the coordination number of $\mathrm{Cl}^{-}$was insensitive to changes in the concentration, but that as the ion concentration increased the coordination number of $\mathrm{Na}^{+}$did drop slightly (to 5.3 at $4.1 \mathrm{M}$ ). The coordination numbers reported in this study (Table 3) fall inside the range of typical values given for both ions. Furthermore, we find no significant change in coordination number over the range of concentrations examined, with the differences lying in the range of the statistical uncertainty.

Solvated FT30 The experimental density of FT30 is measured to be 1.38 $\mathrm{g} \mathrm{cm}^{-3}[5]$ under standard conditions. Density profiles of the three different 
arrangements of systems are shown in Figures 4, 5 and 6 . The density of the water, the FT30 and support and the total density system have been calculated. Defining the membrane bulk as being the central $80.0 \AA$ of the membrane region, then the average system density was calculated as $1.34 \pm 0.01 \mathrm{~g} \mathrm{~cm}^{-3}, 97 \%$ of the experimental value, while the average water density in this region is $0.28 \pm 0.01 \mathrm{~g}$ $\mathrm{cm}^{-3}$. The membrane-bulk solution interface is $\approx 10-20 \AA$ wide, with no sharp transition point. Thus it is difficult to specify the membrane density precisely.

In contrast, the membrane-support interface is quite clearly defined for the non-porous case, P2, with a peak in the density of both the polyamide and the water. Here the polyamide is responsible for the strongly ordered peak immediately adjacent to the support, while the water ordering occurs in the second peak when moving away from the interface. This is consistent with the hydrophobic nature of the description of the coarse-grained polysulfone system. The perturbation of the membrane by the support is found to be reasonable localised at the interface, with little influence on the interface with the solution. The density profile of the pore in run P3 shows an interesting feature; while the total density around the pore is symmetrical, the water density is not, there being a trough on the side of the pore with the membrane. The reason for this is that some of the polyamide chains have entered the mouth of the pore, thus disrupting the sharp interface seen for the non-porous support.

The surface of the membrane at the interface with the solution is found to be rough, commensurate with the thickness observed in the density profiles, with pores, valleys and other features present. This is illustrated in Figure 7 where snapshots of the surface of the membrane in run P2 have been taken at 2 ns intervals. These snapshots also show that that surface of the polyamide membrane has some freedom of movement and therefore dynamically evolves, with some pores closing and new ones opening, with chains shifting position. This non-uniformity might be considered to be a problem for achievement of overall salt-rejection, and indeed leads to variability in the properties of the membrane, as will be demonstrated later.

The overall diffusion coefficient of water within the membrane is $0.21 \pm 0.02 \times$ $10^{-9} \mathrm{~m}^{2} \mathrm{~s}^{-1}$, while Table 4 shows the breakdown of diffusion coefficients for the different systems. These results match well with previous simulations[5], where for a cross-linked polymer at a density of $1.38 \mathrm{~g} \mathrm{~cm}^{-3}$ a diffusion coefficient of $0.20 \times 10^{-9} \mathrm{~m}^{2} \mathrm{~s}^{-1}$ was calculated. While the study of Harder et al. gave a higher diffusion coefficient of $0.50 \times 10^{-9} \mathrm{~m}^{2} \mathrm{~s}^{-1}$ for the water within the membrane, the ratio of the diffusion rate of water within the membrane to that of the bulk was found to be 0.1 , for the TIP3P water model used, similar to the present study. The effect of a support, either porous or non-porous, on the diffusion rate of the water within the membrane is found to be negligible. The water molecules in the membrane form into pockets which are connected to each other by chains of water molecules hydrogen bonded to each other in single file. A typical arrangement is shown in Figure 8. In addition, even when the system has no pressure gradient applied, the diffusion of water molecules both into and out of the membrane is observed. By comparing configurations $1 \mathrm{~ns}$ apart, it is possible to estimate that the average rate of exchange of water molecules between the 
bulk and the membrane is approximately 149 molecules $\mathrm{ns}^{-1}$.

The addition of salt to the bulk solution does not change the above properties particularly; the structure of the membrane remains the same and the diffusion coefficient of water within the membrane is unaltered. As might be expected for a membrane with good salt rejection properties, the salt ions do not show any significant attraction to the membrane surface. There is no congregation of salt ions at the membrane interface and no salt ions enter the membrane during the timescale of the present simulations. Figure 9 shows the density profile for the system without a support; the slight overlap of the polymer density with the salt density is due to the surface roughness of the polymer rather than any salt ions actually entering the membrane. Again the diffusion coefficients of the salt ions in these simulations match well with those of the control runs. The only anomalous result is that for the sodium ion in run P5 where the diffusion coefficient is lower than it should be. This is due to the fact that the ion shows some interaction with the membrane surface (though without ever actually entering the membrane) and is compounded by the fact that at this high dilution the statistics are rather poor. As per the situation for pure water, the presence of the support in the simulation does not influence the results for the saline solutions.

A number of runs with salt ions within the membrane layer were also carried out to examine their properties, should they be able to overcome the barrier to cross the membrane-solution interface. In the first run, $\mathrm{S} 1$, a single $\mathrm{Na}^{+}$and single $\mathrm{Cl}^{-}$were placed in the polyamide, some distance apart. For the second run, S2, the initial coordinates of the $\mathrm{Na}^{+}$were those of the initial coordinates of the $\mathrm{Cl}^{-}$ion in $\mathrm{S} 1$, while the starting position of the $\mathrm{Cl}^{-}$ion in $\mathrm{S} 2$ was the same as the starting position of $\mathrm{Na}^{+}$in $\mathrm{S} 1$ (i.e. the initial positions of the ions in run $\mathrm{S} 2$ were reversed relative to run S1). This attempted to ensure that any effect on the ion due the initial configuration would be able to be observed. For the third run, S3, the concentration of salt ions within the membrane was increased so that seven ions of each species were present in the membrane. Table 4 shows the diffusion coefficients of the ions and water molecules within the membrane.

It appears that the presence of salt ions within the membrane does not change the rate at which water molecules within the membrane diffuse at these salt concentrations. Likewise, the small changes that are seen in the diffusion coefficients of the ions within the membrane with increasing salt concentration are probably nothing more than statistical variation. It does seem that $\mathrm{Cl}^{-}$ diffuses somewhat more slowly within the polyamide than $\mathrm{Na}^{+}$; a result also found by Kotelyanskii et al.[5], and the opposite of the situation in aqueous solutions. Both ions experience at least an order of magnitude reduction in diffusivity within the membrane relative to solution.

Table 5 gives the coordination environment of the salt ions when they are within the solvated FT30. Both ions have their solvation shell disrupted with some of the water molecules being replaced by groups from the membrane. In the case of the sodium ion, at least one water molecule in the solvation shell is replaced by a carbonyl oxygen, while there is also some coordination between the ion and hydroxyl oxygens, but to a lesser degree. The situation with the 
chloride ion is less clear, but at least one, and more usually two, water molecules are removed from the solvation shell, with the ion principally interacting with the hydrogen of the carboxylic acid and the aromatic rings, as well some minor coordination to the amide hydrogens. Kotelyanskii et al. [5] proposed that the sodium ion was coordinated to five water molecules and a carbonyl oxygen while the chloride ion was coordinated to four waters, an amide hydrogen, a carbonyl hydrogen and a phenyl ring. While these results are, on average, similar to those presented in this paper, Kotelyanskii and co-workers proposed that these compositions of the solvation shell were largely fixed, not changing either with time or environment. The results of this study contradict this, with both much more variety in the composition of the solvation shells being observed and with the nature of the coordination environment changing over the course of the simulation. The findings of the present study are supported by the results of solid state ${ }^{23} \mathrm{Na}$ NMR experiments[47] where both a negative chemical shift (suggesting that some of nearest atomic neighbours are polymer atoms) and "significant heterogeneity in the molecular-scale $\mathrm{Na}^{+}$environments" were observed. The cause of the difference in the results of this paper and [5] may simply be due to sampling, as the simulations in the current study are not only longer, but also sample a larger number of salt ions than the previous work.

Free energy surface for ion migration across the membrane-solution interface On the time scale of the present simulations no salt ions were observed entering or leaving the membrane layer. Thus, in order to determine whether this was a result of a thermodynamic barrier or a kinetic barrier, and to gain an idea of the magnitude of any barrier, umbrella sampling methods were used to construct the free energy surface for ions migrating into the membrane from solution.

A set of 40 umbrellas at a spacing of $0.5 \AA$ were set up in the z-axis, spanning from $\approx 7 \AA$ above the membrane surface to a point $\approx 12 \AA$ within the membrane layer. Five runs were carried out for each ion, using different initial $\mathrm{x}$ - and $y$-coordinates, in order to ensure that different parts of the membrane surface were sampled. As no difference is seen in the behaviour of the non-porous support systems and the porous support systems, the former arrangement was used for the umbrella runs as it is computationally cheaper. The solution used in the umbrella sampling runs had a salt concentration of 0.55 molal, with a few salt ions already dispersed within the membrane layer, this setup was used as it most closely resembles that of the real membranes, where there is some diffusion of salt ions into the membrane[47]. The ions within the membrane layer were well distributed, and were well separated from the ions brought across the membrane-solution interface. The duration of these simulations was $550 \mathrm{ps}$ for each umbrella, while the other simulation details are as described in the method section.

The position of the membrane interface is difficult to pinpoint exactly since the membrane surface is not sharply defined and the ion is free to move in the $\mathrm{x}$ and y dimensions. However, the centre point of each umbrella can be used as an 
approximation of the ion position relative to the membrane-solution interface.

As the membrane surface has a relatively large surface area, five umbrella runs were carried out for each ion in order to sample different regions of the interface. Figure 10 shows the free energy profile for the five different runs of an $\mathrm{Na}^{+}$ion entering the membrane. The results cover quite a wide range of energies, but there are some features that seem to be common to most runs. As the ion approaches the membrane there is a slight increase in the free energy, due to the disruption of the solvation shell around the ion. The lack of any sharp barrier at the point where ions enter the membrane indicates the non-uniformity of the membrane surface, with some pathways proving far less energetically unfavourable than others. In all of the runs the largest jump in the free energy profile occurs when the ion is some way into the membrane layer, where the membrane starts to become more dense, and after this peak there is often a local minimum. Figure 11(a) shows the coordination number of the ion to water and carbonyl oxygens as a function of umbrella position for one run; here the disruption of the water solvation as the membrane is approached can be seen clearly. In addition, while the overall coordination number of the sodium is relatively constant, water molecules and carbonyl oxygens exchange with each other in the solvation shell of the ion depending on the local environment. Figure 11(b) shows the number of water molecules coordinated to the ion for three of the different runs (numbers 1, 3 and 4). For run 1, which has a low energy barrier, there are fewer sharp changes in the coordination number compared with the other two runs. This indicates that the height of the energy barrier for the diffusion of the system is linked to the amount of exchange the solvation shell of the ion undergoes as it diffuses into the membrane. Comparing Figure 10 with Figure 11(b) seems to confirm this, since points at which there are sharp changes in composition of the solvation shell of the ion profile coincide with increases in the free energy.

The free energy profile of the $\mathrm{Cl}^{-}$ion, Figure 12, shows both similarities and differences to that of the sodium ion. The free energy required to penetrate a depth of $8 \AA$ into the membrane varies over a relatively large range, $\approx 25-95$ $\mathrm{kJ} \mathrm{mol}{ }^{-1}$. Unlike the case of $\mathrm{Na}^{+}$, however, there does not seem to be any sharp increase in the free energy followed by a local minima. Instead the profiles tend shows a smoother, more gradual increase in free energy, up to a plateau value in two cases. As in the case of the sodium ion, the exchange of water molecules and polymer groups (in this case aromatic rings and the hydroxyl hydrogen) is seen to occur, while the overall coordination number remains relatively constant, as shown in Figure 13(a). This behaviour can also been seen Figure 14, where snapshots have been taken from an umbrella sampling run illustrating the type of diffusion pathway the ions take and how different groups coordinate to the chloride ion as is passes through the membrane-solution interface. Additionally, the same relationship between the magnitude of the free energy barrier and change in water coordination number, Figure 13(b), seen for the sodium ion also applies for the chloride ion; run 3 is the lowest energy pathway and has the smoothest water coordination profile.

It is difficult to determine if the free energy barrier to the diffusion of ions into 
the membrane is greater for one ion than the other from the results obtained as they vary over such a wide range. One major factor in determining the magnitude of the free energy barrier for either ion is the amount of exchange the solvation shell of the ion undergoes as the ion diffuses into the membrane. Runs where there are sudden, large changes in the the solvation shell are the runs that have the higher energy barriers. In turn, this can be correlated with the variable structure of the membrane-solution interface. In some regions there are larger channels of water molecules that permeate the membrane and these obviously represent the preferred pathways for ion diffusion.

A factor that contributes to the energy barrier is the fact that as an single ion is driven to diffuse into the membrane the counter ion is left behind in solution. For the above calculations each ion is assumed to enter the membrane independently, but the energy barrier for the ions to enter the membrane as an ion pair might be lower. Attempts were made to test this theory by drawing an ion pair through the membrane. However, the lifetime of an ion pair is too short, with the ion pairs dissociating before they can enter the membrane. In addition, when a ion is pulled into the membrane no diffusion of any counter ion into the membrane is observed.

Although it is difficult to determine the free energy for an ion pair to enter the membrane, it is possible, however, to determine the free energy profile of a $\mathrm{Cl}^{-}$ion entering the membrane after a sodium ion has already diffused into the membrane. This was done by taking, from three of the $\mathrm{Na}^{+}$umbrella sampling runs (1,3 and 4), configurations where the sodium ion was in a local energy minimum, and then performing further umbrella sampling runs on a chloride ion, to compute the free energy profile to bring this ion to the same depth into the membrane as the sodium ion minimum. The results of these three umbrella simulations are shown in Figure 15. The free energy profiles for these three runs fall within the range of energy values generated for a chloride ion entering the membrane without any corresponding sodium ion and are consistent with those results. However, what is surprising is that the presence of the sodium ion within the membrane does not appear to assist in the diffusion of $\mathrm{Cl}^{-}$ions into the membrane, another indication that the membrane local environment is the dominant factor in the free energy barrier.

\section{Conclusions}

Molecular dynamics simulations of the FT30 polyamide reverse osmosis membrane have been carried out in order to determine the detailed properties of this system, at the atomic level, relevant to desalination. Diffusion coefficients of the species in both the bulk solution and within the membrane layer itself have been calculated, showing that water diffuses approximately an order of magnitude slower within the membrane layer than in bulk solution. The presence of a support, either porous or non-porous, had a negligible effect in these simulations where there is no pressure difference across the system. The diffusion of $\mathrm{Na}^{+}$ and $\mathrm{Cl}^{-}$ions in polyamide is slower than water, with the reduction relative to so- 
lution being similar. However, the diffusion of chloride ions is slowed to a greater extent than for sodium, leading to a reversal in the relative diffusion rates in comparison to saline solution. The observation that the rates of diffusion for all species are decreased by approximately the same factor has implications for the design of more efficient membranes based on the polyamide system. To reduce the energy consumption, the ideal situation would be to increase the flow rate of water for a given pressure, while maintaining the salt-rejection at the same level. If the diffusivities of all species are similarly influenced by the membrane structure, then the selectivity may remain constant even at higher flow rates, thus making it feasible to reduce the energetic cost.

How the ions interact with the polyamide has been investigated by determination of the coordination numbers of the ions with various groups within the membrane. Even when the ions are within the membrane, water molecules account for over $50 \%$ of the species coordinated to the ions. From the polymer, $\mathrm{Na}^{+}$coordinates most strongly with the carbonyl oxygen, and shows a lesser degree of interaction with the hydroxyl oxygen. The chloride ion loses a greater percentage of water molecules from its solvation shell, and shows some coordination with the hydroxyl hydrogen, the aromatic rings and the amide hydrogens. The greater coordination of polymer groups to the $\mathrm{Cl}^{-}$ion is likely to be the reason for the greater relative reduction of the diffusion coefficient of the chloride ion compared to the sodium ion within the membrane.

In order to investigate the behaviour at the membrane interface a number of runs were carried out using umbrella sampling methods to determine the free energy surface associated with an ion diffusing into the polyamide. The results show that the rate of increase in free energy varies quite considerably, making it difficult to determine if one ion would find it easier to enter the membrane than the other. One result seen in every run was that even before the ion entered the membrane the disruption of the solvation shell around the ion resulted in an energetic penalty. In addition, the profiles did not show a sharp peak in the free energy at the membrane interface but a more gradual increase as the ion diffused into the membrane. The size of the free energy increase partly depends upon the degree of disruption of the solvation shell that an ion undergoes, with the higher energy pathways showing both more frequent exchanges of the species coordinated to the ions and a greater degree of change in the composition of the solvation shell at any one time.

Although the free energy profiles for both sodium and chloride diffusion into the membrane show considerable variability, corresponding to the different range of environments possible within FT-30, there are ultimately no facile pathways through the approximately $100 \AA$ thick membrane used in the present simulations. Given that the experimental membrane used is typically twenty times this thickness, this will be even more true of the real material. Attempts to force an ion to migrate through the membrane show an increasing free energy profile with no signs of a plateau, at least to a depth of $10 \AA$. This behaviour can be explained in terms of the increasing Coulomb energy of separation of the ion and the counter-balancing charge remaining above the interface, with a variable effective dielectric constant depending on the water structure in the membrane 
and its ability to reorient to screen the interaction. It might be expected that this Coulomb interaction would reach the point where it is strong enough to allow the counter ion to also enter the membrane itself. However, this is not observed in any of the present simulations. Even when using umbrella sampling to examine the free energy to bring a chloride anion toward a net sodium ion that already resides within the membrane, there remains a significant resistance to creating a separated ion pair. This finding is important since it suggests the absence of a low energy pathway for sodium chloride through the less hydrophilic membrane by ion pairing.

Molecular dynamic studies can provide information about RO membranes that is difficult, or even impossible, to determine by experimental methods. The simulations carried out in this work describe the behaviour of water molecules, sodium cations and chloride anions with the FT30 membrane under standard conditions. This allows the results of future simulations investigating changes to the membrane structure, interactions with foulant species and the behaviour of the membrane under pressure to be evaluated properly. Combining the data obtained from simulation with that obtained by experimental methods will allow new more efficient membranes in be developed.

\section{Acknowledgments}

This research was supported by the Australian Commonwealth Scientific and Research Organisation (CSIRO) under the "Water For A Healthy Country" Flagship. Additionally the authors would like to thank the cluster members, especially Stephen Grey, for their comments and discussion. JDG would like to thank the Australian Research Council (ARC) for a professorial fellowship. iVEC and the National Computational Infrastructure (NCI) provided the computational resources needed to carry out this work.

\section{References}

[1] Fritzmann, C.; Loewenberg, J.; Wintgens, T.; Melin, T. Desalination 2007, $216,1-76$.

[2] Khawaji, A. D.; Kutubkhanah, I. K.; Wie, J.-M. Desalination 2008, 221, 47-69.

[3] Charcosset, C. Desalination 2009, 245, 214-231.

[4] Saliby, I. E.; Okour, Y.; Shon, H. K.; Kandasamy, J.; Kim, I. S. Desalination 2009, 247, 1-14.

[5] Kotelyanskii, M. J.; Wagner, N. J.; Paulaitis, M. E. J. Mem. Sci. 1998, 139, 1-16.

[6] Khedr, M. G. Desalination 2003, 153, 295-304. 
[7] Mi, B.; Coronell, O.; Marinas, B. J.; Watanabe, F.; Cahill, D. G.; Petrov, I. J. Mem. Sci. 2006, 282, 71-81.

[8] Singh, P. S.; Joshi, S. V.; Trivedi, J. J.; Devmurari, C. V.; Rao, A. P.; Ghosh, P. K. J. Mem. Sci. 2006, 278, 19-25.

[9] Mi, B.; Cahill, D. G.; Marinas, B. J. J. Mem. Sci. 2007, 291, 77-85.

[10] Murad, S.; Lin, J. Chem. Eng. J. 1999, 74, 99-108.

[11] Murad, S.; Nitsche, L. C. Chem. Phys. Lett. 2004, 397, 211-215.

[12] Qiao, R.; Georgiadis, J. G.; Aluru, N. R. Nano. Lett. 2006, 6, 995-999.

[13] Raghunathan, A. V.; Aluru, N. R. Phys. Rev. Lett. 2006, 97, 024501.

[14] Harder, E.; Walters, D. E.; Bodnar, Y. D.; Faibish, R. S.; Roux, B. J. Phys. Chem. B 2009, 113, 10177-10182.

[15] Cadotte, J. E.; Petersen, R. J.; Larson, R. E.; Erickson, E. E. Desalination 1980, 32, 25-31.

[16] Petersen, R. J. J. Mem. Sci 1993, 83, 81-150.

[17] Jorgensen, W. L.; Tirado-Rives, J. J. Am. Chem. Soc. 1988, 110, 16571666.

[18] Jorgensen, W. L.; Maxwell, D. S.; Tirado-Rives, J. J. Am. Chem. Soc. 1996, 118, 11225-11236.

[19] Rizzo, R. C.; Jorgensen, W. L. J. Am. Chem. Soc. 1999, 121, 4827-4836.

[20] Damm, W.; Gunsteren, W. E. V. J. Comput. Chem. 2000, 21, 774-787.

[21] Anderson, P. M.; Wilson, M. R. J. Chem. Phys. 2004, 121, 8503-8510.

[22] Snow, C. D.; Qiu, L. L.; Du, D. G.; Gai, F.; Hagen, S. J.; Pande, V. S. PNAS 2004, 101, 4077-4082.

[23] Adisa, B.; Bruce, D. J .Phys. Chem. B 2005, 109, 7548-7556.

[24] Tieleman, D. P.; MacCallum, J. L.; Ash, W. L.; Kandt, C.; Xu, Z. T.; Monticelli, L. J. Phys. Condens. Mat. 2006, 18, S1221-S1234.

[25] Daub, C. D.; Leung, K.; Luzar, A. J. Phys. Chem. B 2009, 113, 7687-7700.

[26] Horn, H. W.; Swope, W. C.; Pitera, J. W.; Madura, J. D.; Dick, T. J.; Hura, G. L.; Head-Gordon, T. J. Chem. Phys. 2004, 120, 9665-9678.

[27] Joung, I. S.; Cheatham, T. E. J. Phys. Chem. B 2008, 112, 9020-9041.

[28] Joung, I. S.; Cheatham, T. E. J. Phys. Chem. B 2009, 113, 13279-13290. 
[29] Smith, W.; Forester, T. R. J. Mol. Graphics 1996, 14, 136-141.

[30] Essmann, U.; Perera, L.; Berkowitz, M. L.; Darden, T.; Lee, H.; Pedersen, L. G. J. Chem. Phys. 1995, 103, 8577-8593.

[31] Nose, S. J Chem. Phys. 1984, 81, 511-519.

[32] Hoover, W. G. Phys. Rev. A 1985, 31, 1695-1697.

[33] Bonomi, M.; Branduardi, D.; Bussi, G.; Camilloni, C.; Provasi, D.; Raiteri, P.; Donadio, D.; Marinelli, F.; Pietrucci, F.; Broglia, R. A.; Parrinello, M. Comput. Phys. Commun. 2009, 180, 1961-1972.

[34] Patra, M.; Karttunen, M. J. Comput. Chem. 2004, 25, 678-689.

[35] Lau, K. F.; Alper, H. E.; Thacher, T. S.; Stouch, T. R. J. Phys. Chem. 1994, 98, 8785-8792.

[36] Mahoney, M. W.; Jorgensen, W. L. J. Chem. Phys. 2001, 114, 363-366.

[37] Kotelyanskii, M. J.; Wagner, N. J.; Paulaitis, M. E. Comput. Theor. Polym. S. 1999, 9, 301-306.

[38] Marcus, Y. Ion Properties; Marcel Dekker: New York, 1997.

[39] Woolf, L. A. J. Phys. Chem. 1978, 82, 959-962.

[40] Lyubartsev, A. P.; Laaksonen, A. J. Phys. Chem. 1996, 100, 16410-16418.

[41] Toukan, K.; Rahman, A. Phys. Rev. B 1985, 31, 2643-2648.

[42] Dang, L. X.; Smith, D. E. J. Chem. Phys. 1993, 99, 6950-6956.

[43] Smith, D. E.; Dang, L. X. J. Chem. Phys. 1994, 100, 3757-3766.

[44] Muller, K. J.; Hertz, H. G. J. Phys. Chem. 1996, 100, 1256-1265.

[45] Uchida, H.; Matsuoka, M. Fluid Phase Equil. 2004.

[46] Koneshan, S.; Rasaiah, J. C. J. Chem. Phys. 2000, 113, 8125-8137.

[47] Xu, X.; Kirkpatrick, R. J. J Mem. Sci. 2006, 280, 226-233. 


\begin{tabular}{c|cccc|ccc} 
Run & $\begin{array}{c}\text { Support } \\
\text { present }\end{array}$ & $\begin{array}{c}\text { Pore } \\
\text { present }\end{array}$ & $\begin{array}{c}\text { Membrane } \\
\text { present }\end{array}$ & $\begin{array}{c}\text { Salt inside } \\
\text { membran }\end{array}$ & {$[\mathrm{NaCl}] /$ molal } & $\begin{array}{c}\text { Number } \\
\text { of NaCl }\end{array}$ & $\begin{array}{c}\text { Duration of } \\
\text { production run/ns }\end{array}$ \\
\hline C1 & no & no & no & N/A & - & 0 & 2 \\
C2 & no & no & no & N/A & 0.0055 & 1 & 2 \\
C3 & no & no & no & N/A & 0.55 & 100 & 2 \\
\hline P1 & no & no & yes & N/A & - & 0 & 6 \\
P2 & yes & no & yes & N/A & - & 0 & 10 \\
P3 & yes & yes & yes & N/A & - & 0 & 8 \\
P4 & yes & no & yes & no & 0.0055 & 1 & 8 \\
P5 & yes & yes & yes & no & 0.0055 & 1 & 8 \\
P6 & yes & no & yes & no & 0.55 & 100 & 6 \\
P7 & yes & yes & yes & no & 0.55 & 100 & 6 \\
P8 & no & no & yes & no & 0.55 & 100 & 8 \\
\hline S1 & yes & no & yes & yes & 0.0055 & 1 & 6 \\
S2 & yes & no & yes & yes & 0.0055 & 1 & 6 \\
S2 & yes & no & yes & yes & 0.55 & 100 & 6
\end{tabular}

Table 1: Details of the different simulations performed in the present study of the FT30 desalination membrane. 9828 water molecules were present in all the runs except P3, P4 and P7 where 16055 were present. 


\begin{tabular}{c|c|c|c} 
Run (salt concentration/molal) & $D_{\text {water }} / 10^{-9} \mathrm{~m}^{2} \mathrm{~s}^{-1}$ & $D_{\mathrm{Na}+} / 10^{-9} \mathrm{~m}^{2} \mathrm{~s}^{-1}$ & $D_{C l} / 10^{-9} \mathrm{~m}^{2} \mathrm{~s}^{-1}$ \\
\hline C1 $(0.0)$ & $2.70 \pm 0.03$ & - & - \\
C2 $(0.0055)$ & $2.71 \pm 0.03$ & $1.51 \pm 0.40$ & $2.07 \pm 0.60$ \\
C3 (0.55) & $2.53 \pm 0.03$ & $1.22 \pm 0.02$ & $1.67 \pm 0.03$ \\
\hline Experiment ( $\infty$ dilution) [38] & 2.35 & 1.33 & 2.03 \\
Experiment (0.5M)[39] & 2.27 & 1.28 & 1.85 \\
TIP4P-Ew (1.0)[28] & - & 1.12 & 1.52 \\
SPC-Fw (0.55)[40] & 2.05 & 0.93 & 1.27
\end{tabular}

Table 2: Diffusion coefficients of water, sodium cations and chloride anions as determined from the control runs carried out in this study. Literature values are included for comparison. 


\begin{tabular}{c|c|c} 
Run (salt concentration / molal) & $\mathrm{Na}^{+}$coord. number & $\mathrm{Cl}^{-}$coord. number \\
\hline C2 $(0.0055)$ & $6.05 \pm 0.02$ & $6.80 \pm 0.02$ \\
C3 $(0.55)$ & $6.00 \pm 0.05$ & $6.82 \pm 0.03$
\end{tabular}

Table 3: Average coordination numbers of the sodium and chloride ions determined from the control runs. 


\begin{tabular}{c|cccccc} 
& \multicolumn{6}{|c}{ Diffusion coefficient $/ 10^{-9} \mathrm{~m}^{2} \mathrm{~s}^{-1}$} \\
$\begin{array}{c}\text { Simulation } \\
\text { run }\end{array}$ & Water & In solution & $\mathrm{Cl}$ & Water & $\mathrm{Na}$ & $\mathrm{Cl}$ \\
\hline P1 & $2.71 \pm 0.07$ & - & - & $0.18 \pm 0.02$ & - & - \\
P2 & $2.73 \pm 0.07$ & - & - & $0.22 \pm 0.02$ & - & - \\
P3 & $2.64 \pm 0.08$ & - & - & $0.23 \pm 0.04$ & - & - \\
P4 & $2.76 \pm 0.07$ & $1.41 \pm 0.48$ & $2.10 \pm 0.66$ & $0.23 \pm 0.02$ & - & - \\
P5 & $2.66 \pm 0.09$ & $1.05 \pm 0.23$ & $1.93 \pm 0.48$ & $0.24 \pm 0.05$ & - & - \\
P6 & $2.46 \pm 0.06$ & $1.20 \pm 0.03$ & $1.61 \pm 0.03$ & $0.20 \pm 0.01$ & - & - \\
P7 & $2.55 \pm 0.07$ & $1.28 \pm 0.06$ & $1.75 \pm 0.07$ & $0.23 \pm 0.03$ & - & - \\
P8 & $2.49 \pm 0.05$ & $1.21 \pm 0.09$ & $1.58 \pm 0.05$ & $0.17 \pm 0.01$ & - & - \\
S1 & $2.74 \pm 0.06$ & - & - & $0.23 \pm 0.02$ & $0.10 \pm 0.04$ & $0.07 \pm 0.02$ \\
S2 & $2.69 \pm 0.07$ & - & - & $0.22 \pm 0.02$ & $0.10 \pm 0.03$ & $0.07 \pm 0.02$ \\
S3 & $2.54 \pm 0.12$ & $1.32 \pm 0.13$ & $1.76 \pm 0.14$ & $0.19 \pm 0.01$ & $0.14 \pm 0.03$ & $0.04 \pm 0.01$
\end{tabular}

Table 4: Diffusion coefficients of water, $\mathrm{Na}^{+}$and $\mathrm{Cl}^{-}$within the membrane and solution, taken from the different simulation runs. 


\begin{tabular}{c|c|ccccc} 
Species & Run & \multicolumn{6}{|c}{ Coordination number } \\
& & Water & Carbonyl & Hydroxyl & Aromatic ring & Amide \\
\hline $\mathrm{Na}$ & S1 & $4.02 \pm 0.60$ & $2.09 \pm 0.62$ & $0.01 \pm 0.03$ & - & - \\
& S2 & $5.19 \pm 0.24$ & $0.74 \pm 0.22$ & $0.21 \pm 0.14$ & - & - \\
& S3 & $4.58 \pm 0.29$ & $1.28 \pm 0.21$ & $0.24 \pm 0.15$ & - & - \\
\hline $\mathrm{Cl}$ & S1 & $3.97 \pm 1.15$ & - & $1.62 \pm 0.52$ & $0.65 \pm 0.28$ & $0.30 \pm 0.26$ \\
& S2 & $6.15 \pm 0.20$ & - & $0.03 \pm 0.03$ & $0.22 \pm 0.16$ & $0.03 \pm 0.03$ \\
& S3 & $4.82 \pm 0.38$ & - & $0.84 \pm 0.23$ & $0.71 \pm 0.30$ & $0.26 \pm 0.15$
\end{tabular}

Table 5: Coordination numbers of ions to different groups when within the the FT30 membrane. 


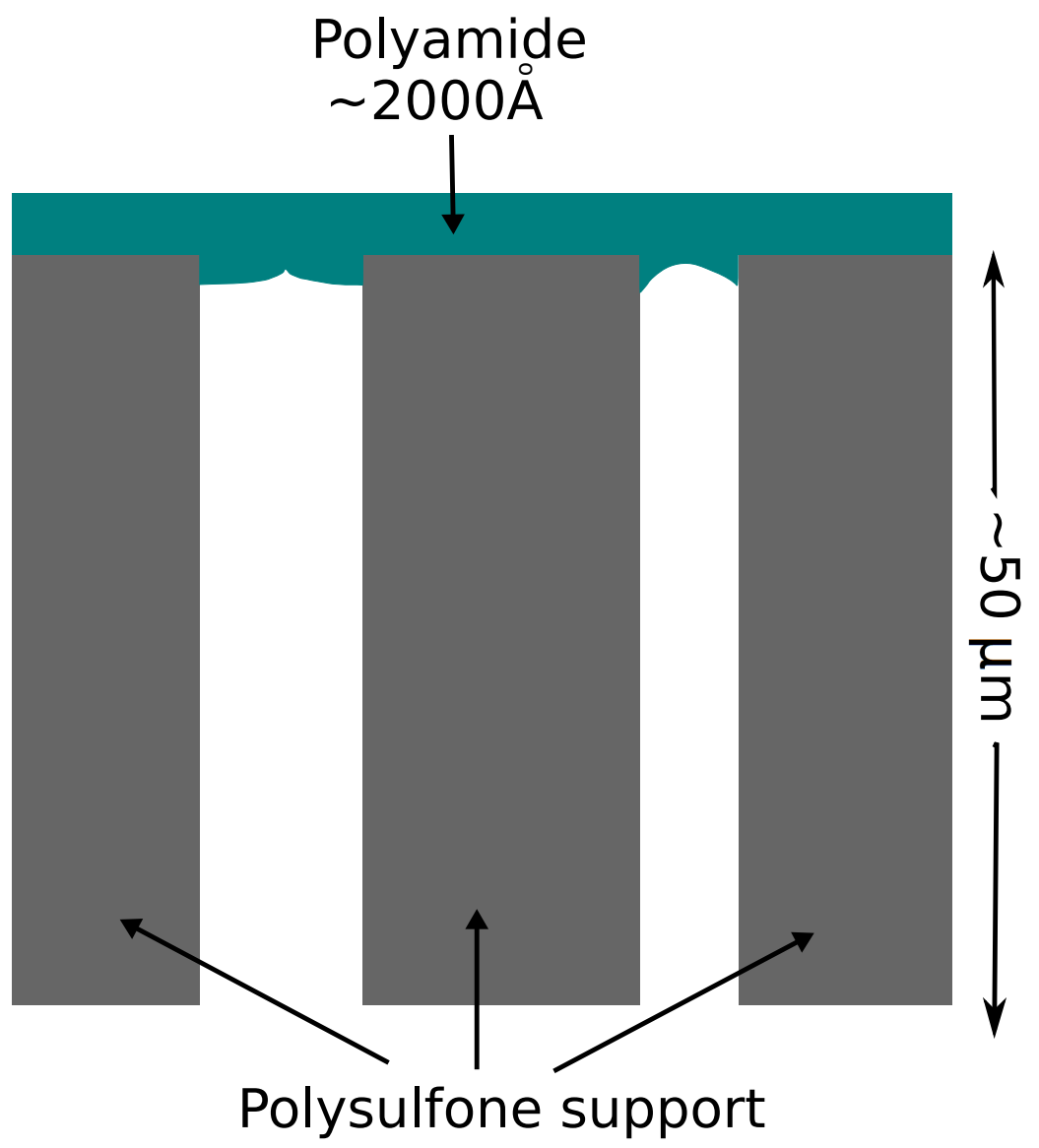

Figure 1: Schematic diagram of the polyamide-polysulfone composite membranes currently used in reverse osmosis. Distances indicated represent approximate the thickness of the relevant layer 


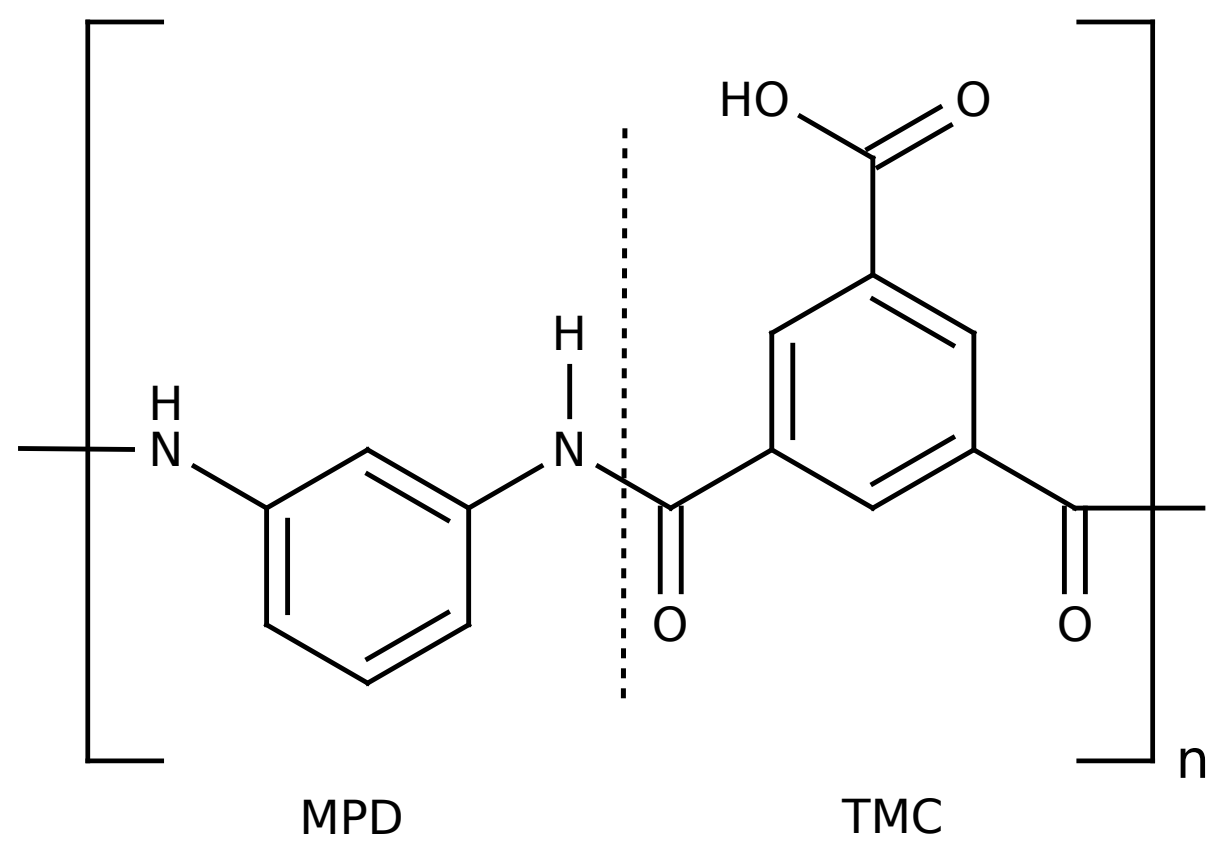

Figure 2: Schematic representation of the structure of the polyamide FT30, illustrating the two monomers, TMC and MPD, that compose a repeat unit. 

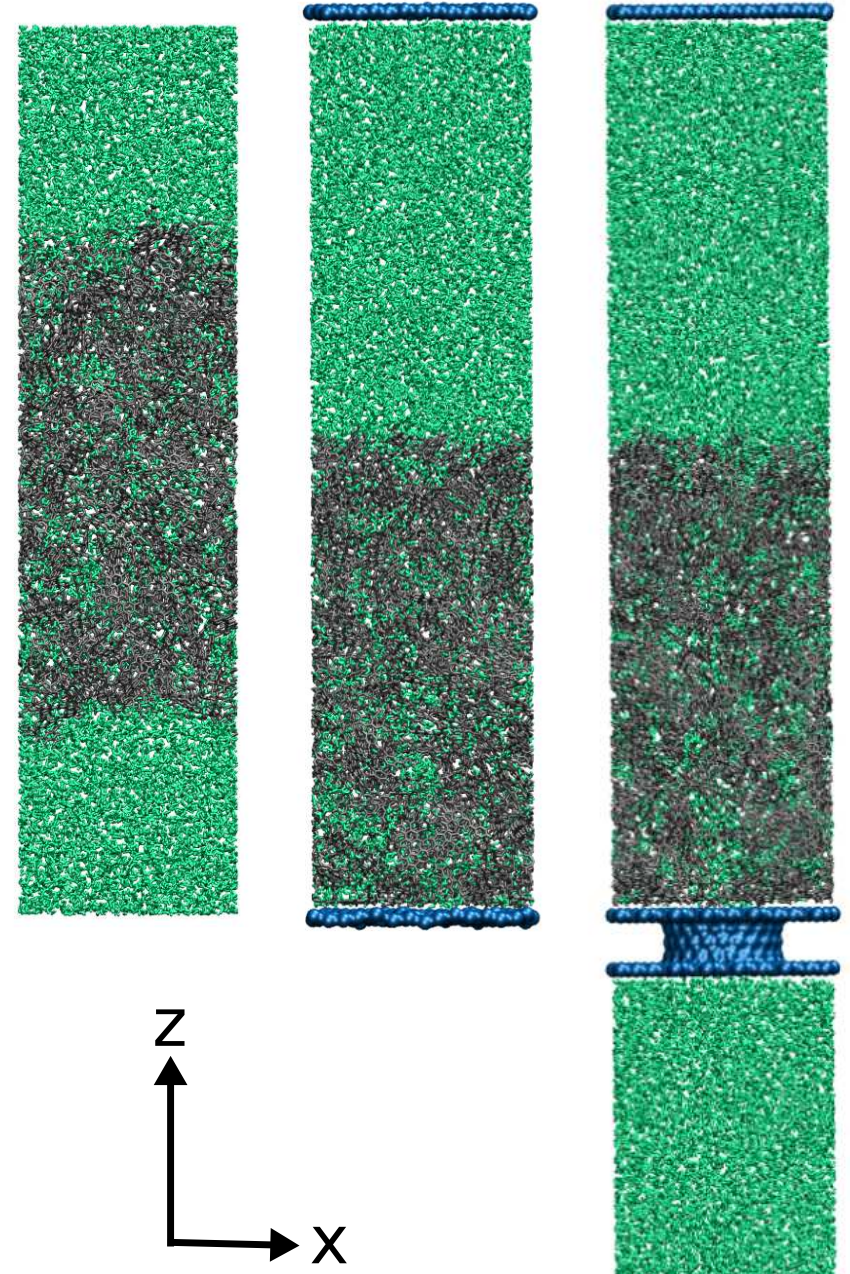

Figure 3: The three different system arrangements simulated in the present work; (left to right) a system without any support, a system with a non-porous support layer, and a system with a porous support. The water is coloured green, the polyamide grey, and the coarse-grained polysulfone support blue. 

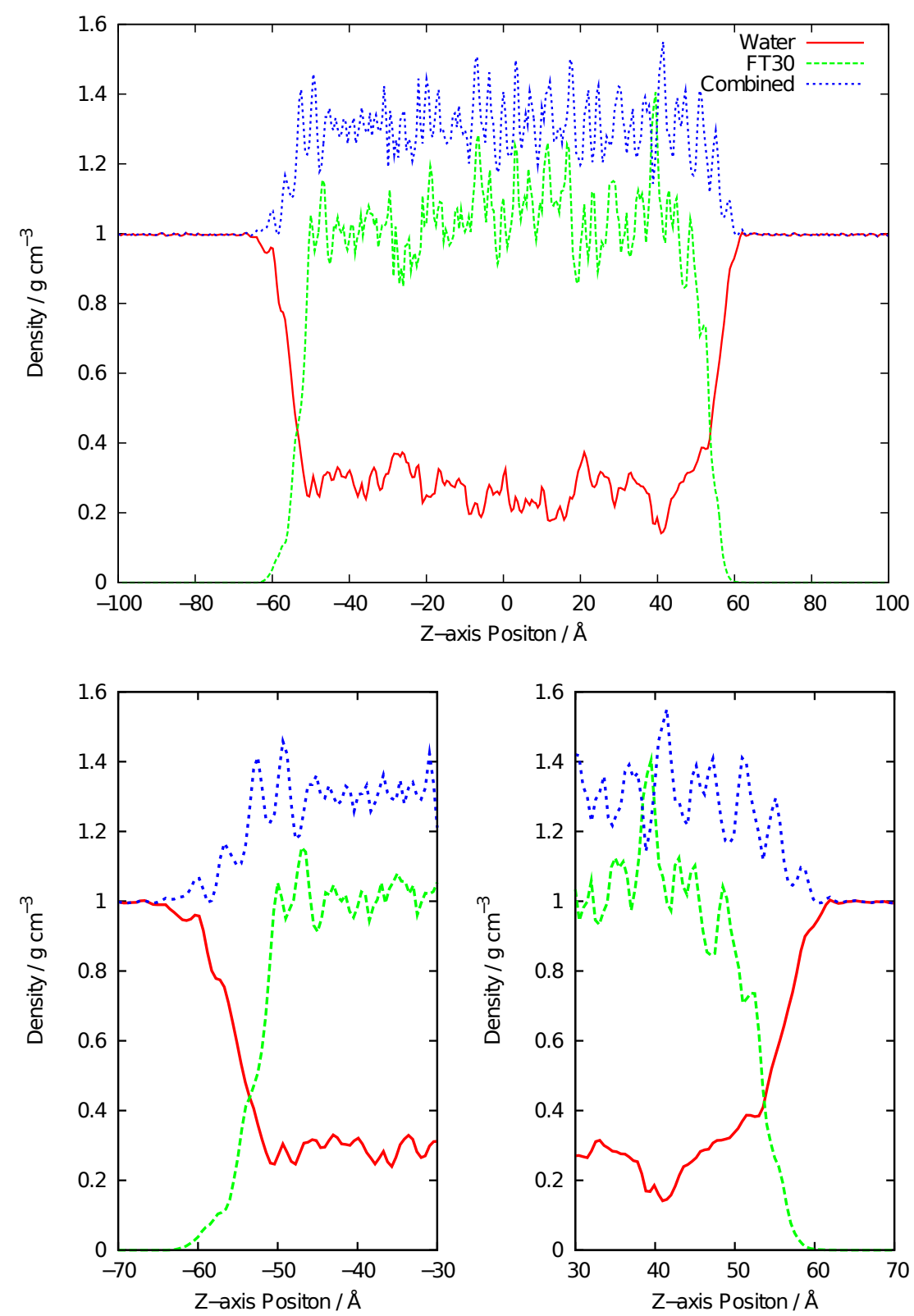

Figure 4: Density profiles of FT30 membrane taken from run P1, (a) the full system, (b) and (c) closeups of the profiles at the membrane-bulk solution interfaces. 

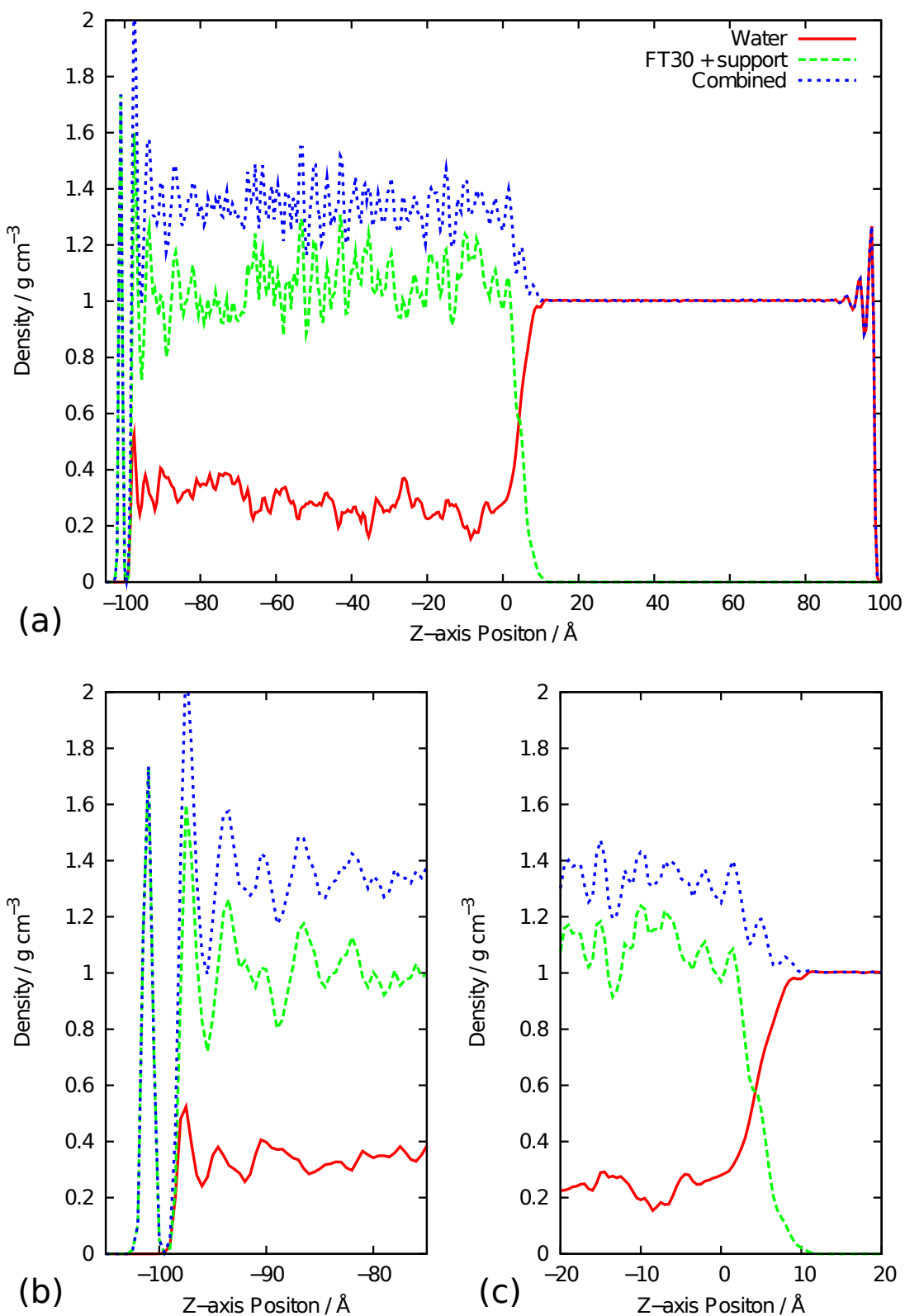

Figure 5: Density profiles of FT30 membrane taken from run P2, (a) the full system, (b) the membrane-bulk solution interface and (c) the membrane-support interface. 

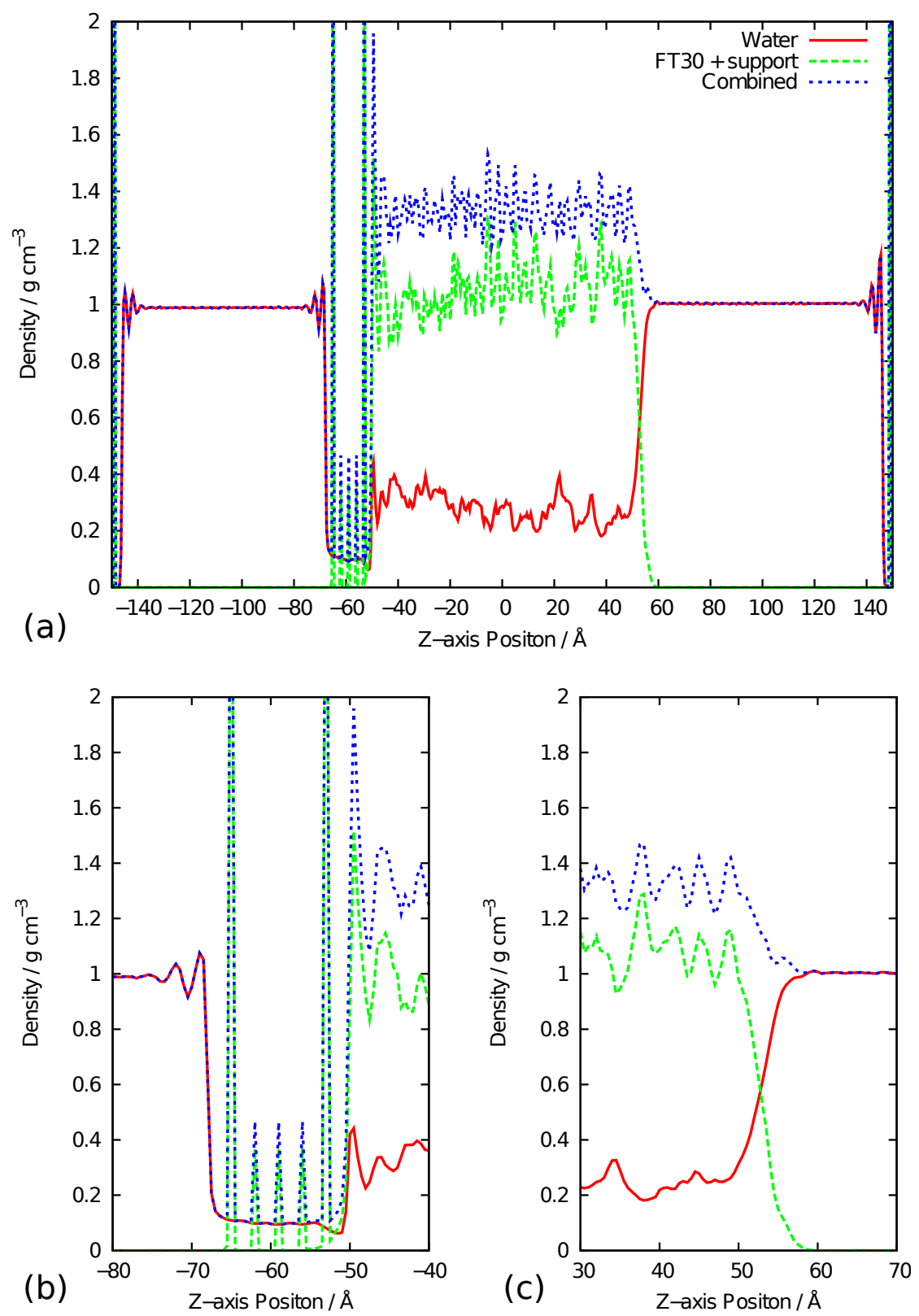

Figure 6: Density profiles of FT30 membrane taken from run P3, (a) the full system, (b) the membrane-bulk solution interface and (c) the membrane-porous support interface. 


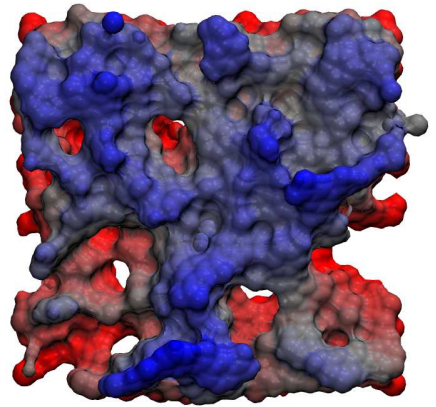

(a)

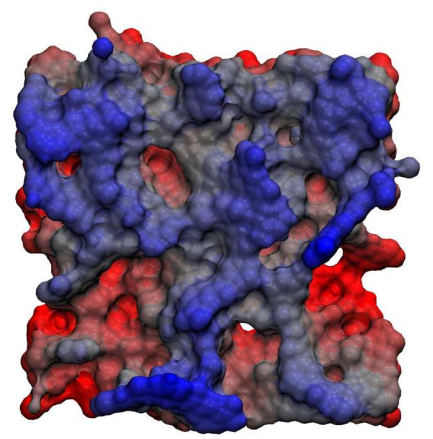

(c)

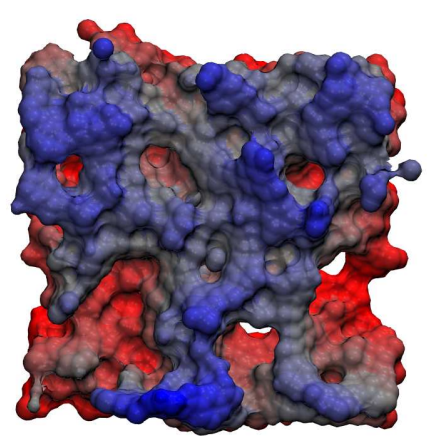

(e)

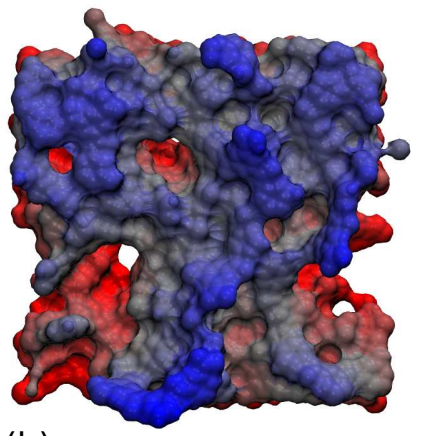

(b)

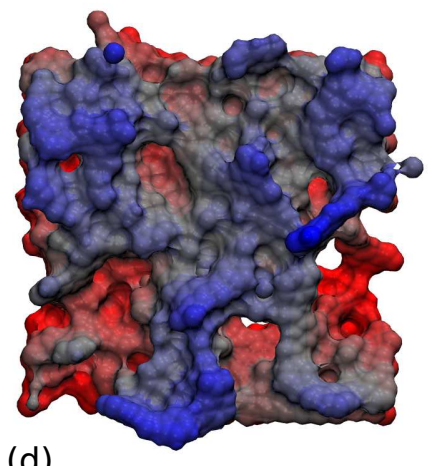

(d)

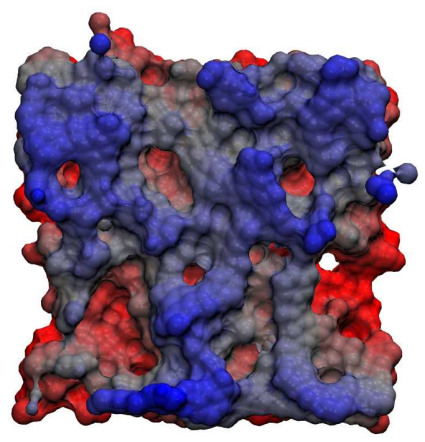

(f)

Figure 7: Snapshots of the surface the membrane forms with the bulk solution taken from run P2 looking down the z-axis at (a) 0 ns, (b) 2 ns, (c) 4 ns, (d) 6 ns, (e) $8 \mathrm{~ns}$ and (f) $10 \mathrm{~ns}$. Water molecules have been removed for clarity and the colouring depends on the z-coordinates of the atoms, red $=$ bottom, grey $=$ middle, blue $=$ top, where the $\mathrm{z}$-coordinate becomes increasingly positive when moving from the membrane into solution. The snapshots illustrate both that the membrane-water interface shows atomic roughness and some degree of fluidity. 


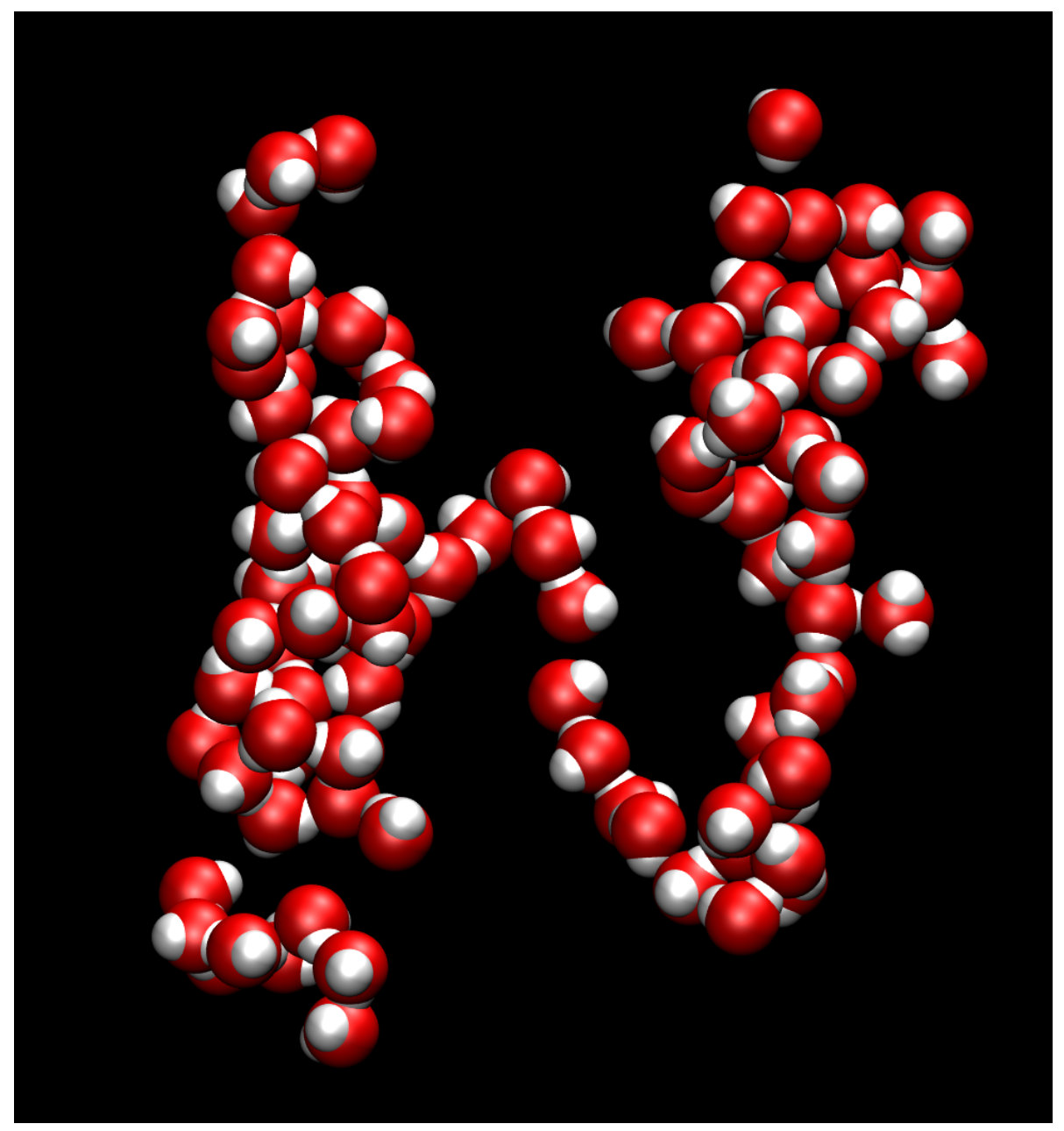

Figure 8: Snapshot taken from a simulation illustrating the water structure inside the membrane, consisting of clusters connected by linear chains of water molecules. Here oxygen and hydrogen are shown in red and white, respectively, while the polyamide is omitted for clarity. 


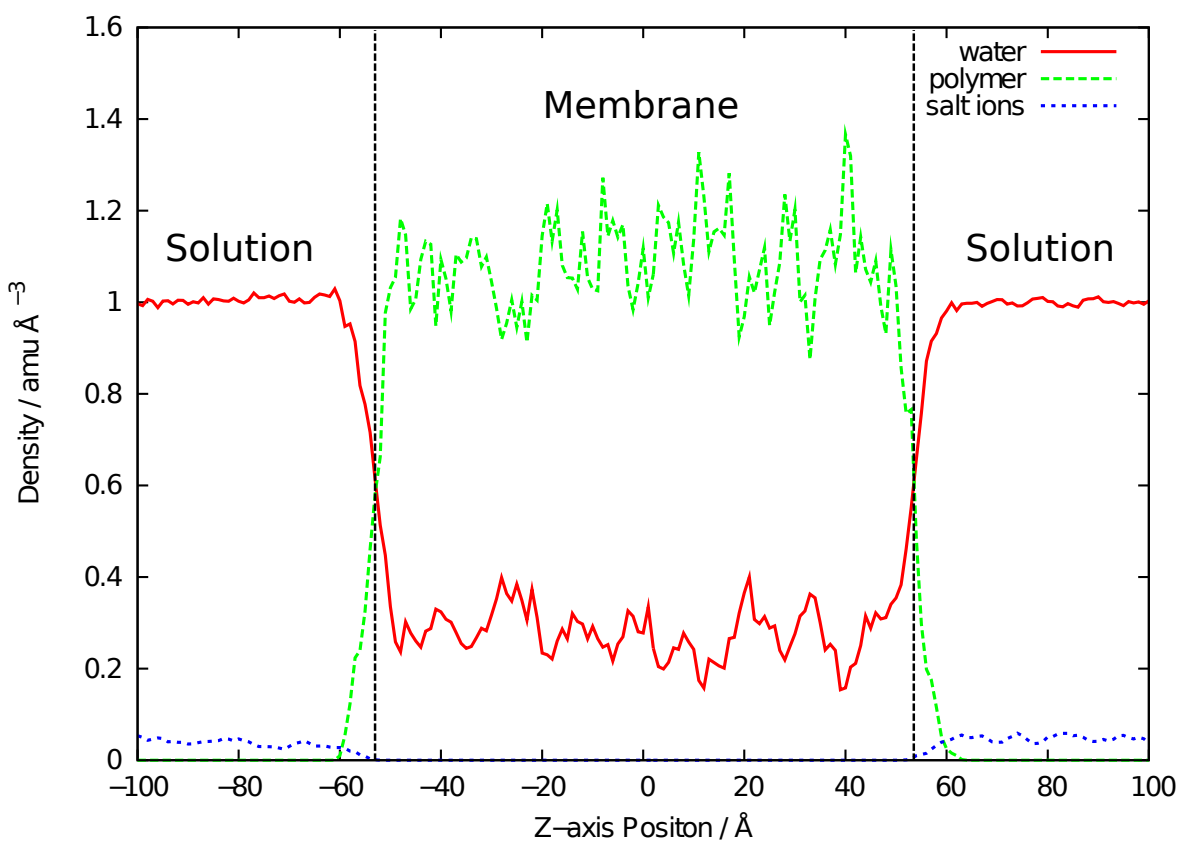

Figure 9: Density profile of FT30 membrane in contact with seawater $(0.55$ molal $\mathrm{NaCl}$, run $\mathrm{P} 8$ ). 


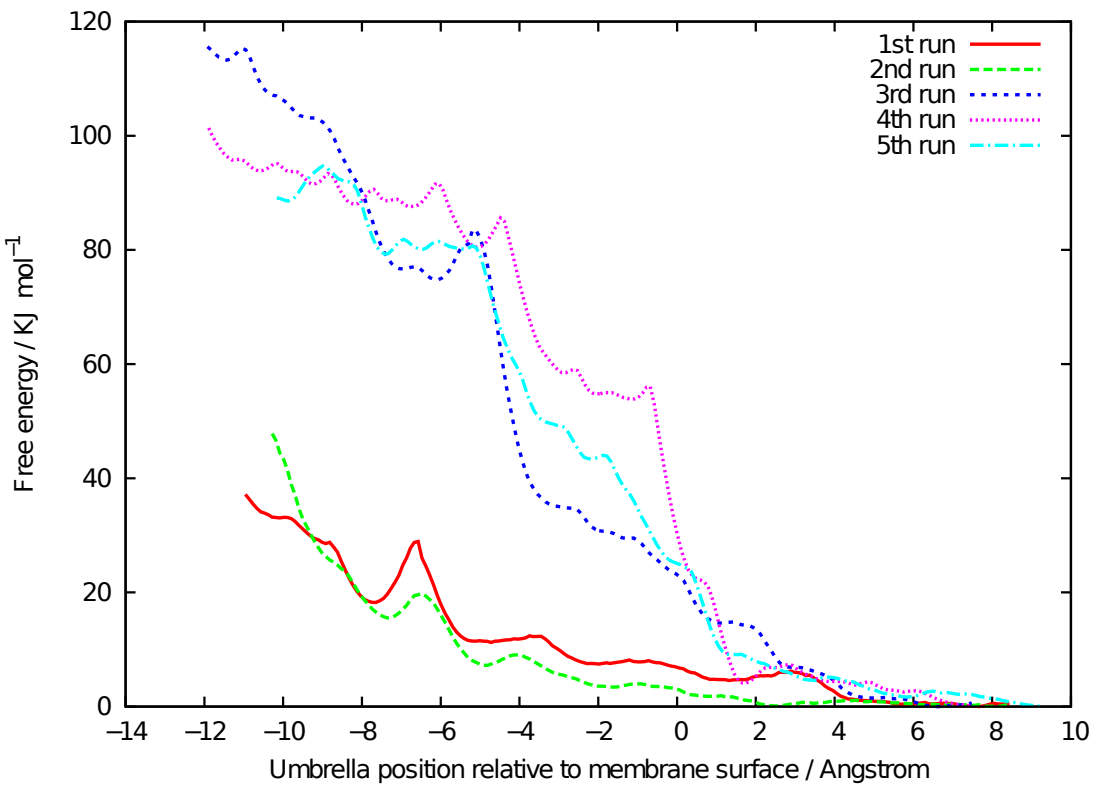

Figure 10: Free energy profile for a $\mathrm{Na}^{+}$ion to enter the FT30 membrane determined using umbrella sampling methods. An increasing negative position indicates the ion is moving into the membrane. 

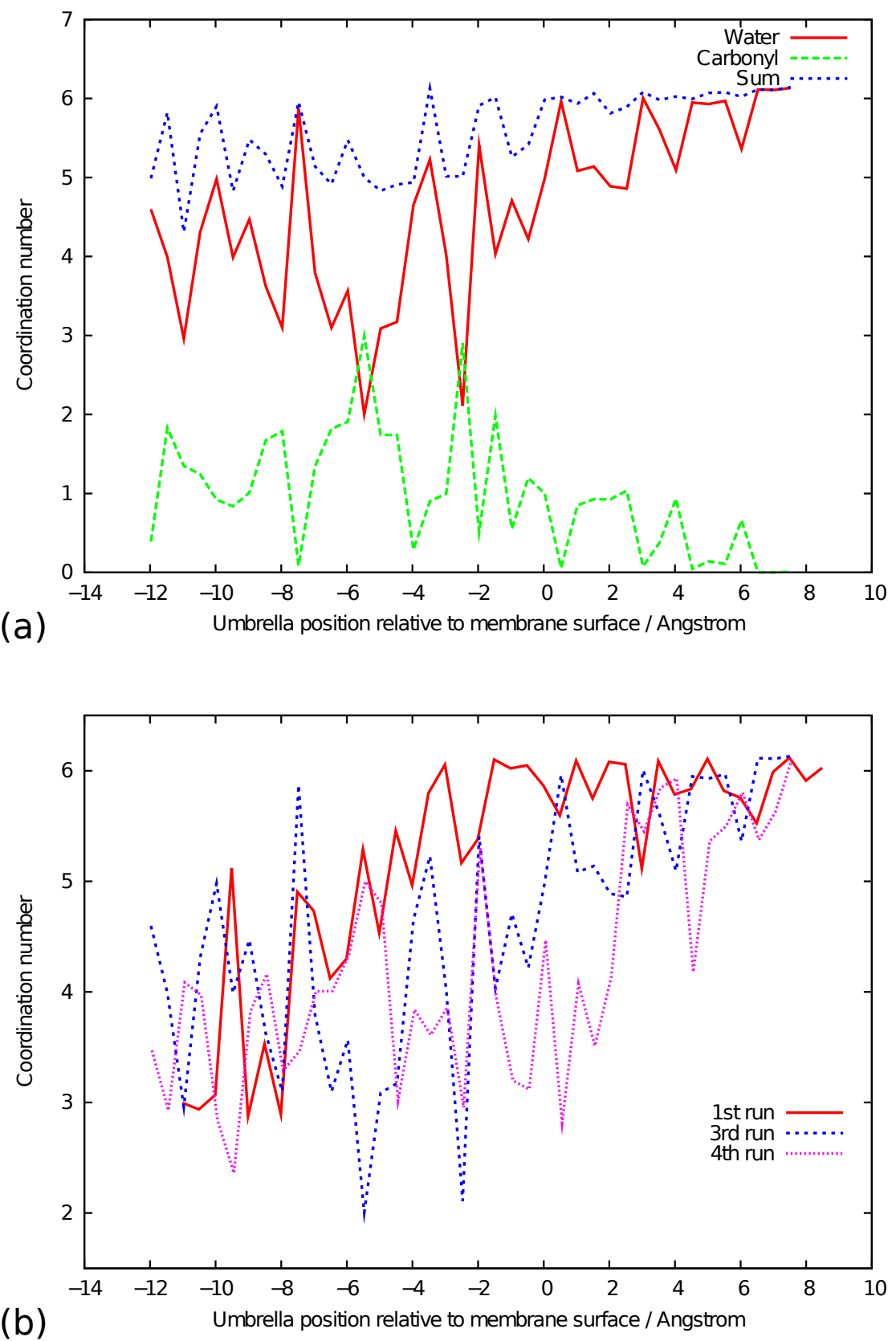

Figure 11: Coordination number of $\mathrm{Na}^{+}$ion as a function of the umbrella position. (a) shows the coordination number of the sodium ion to water molecules, carbonyl oxygens and the overall coordination number for the $\mathrm{Na}^{+}$umbrella sampling run 3. (b) shows the coordination of the sodium ion to water molecules in runs 1,3 and 4 . 


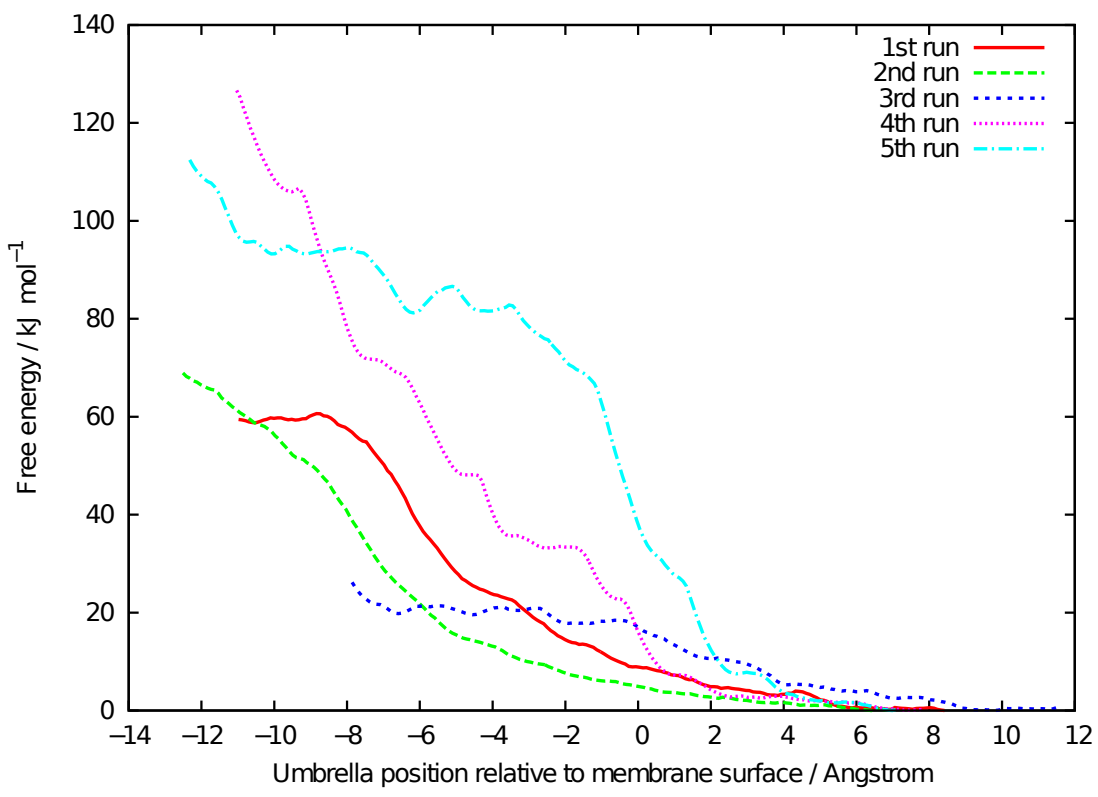

Figure 12: Free energy profile for a $\mathrm{Cl}^{-}$ion to enter the FT30 membrane determined using umbrella sampling methods. An increasing negative position indicates the ion is moving into the membrane. 

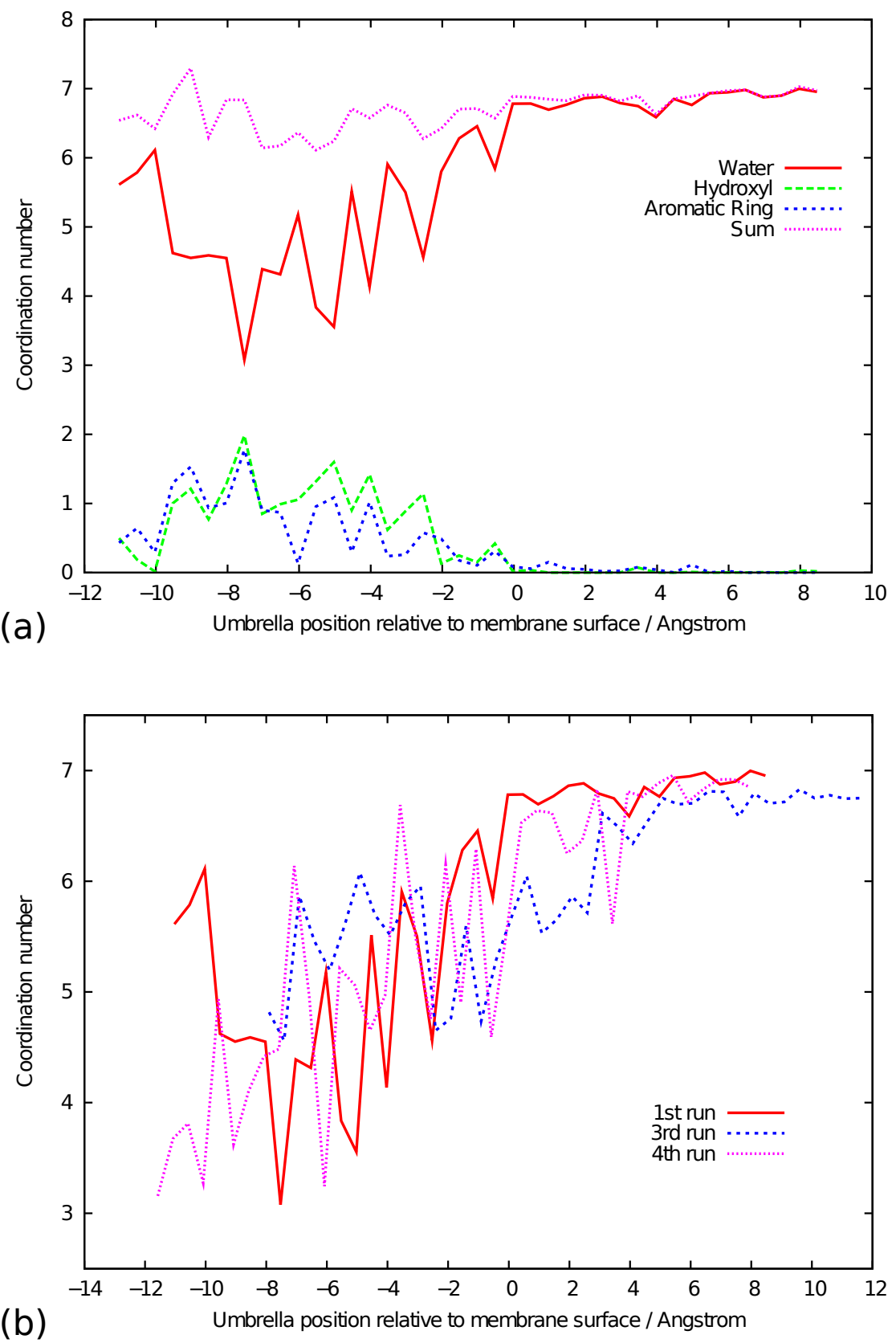

Figure 13: Coordination number of $\mathrm{Cl}^{-}$ion as a function of the umbrella position. (a) shows the coordination number of the chloride ion to water molecules, hydroxyl groups, aromatic rings and the overall coordination number for the $\mathrm{Cl}^{-}$umbrella sampling run 1 . (b) shows the coordination of the chloride ion to water molecules in runs 1,3 and 4 . 


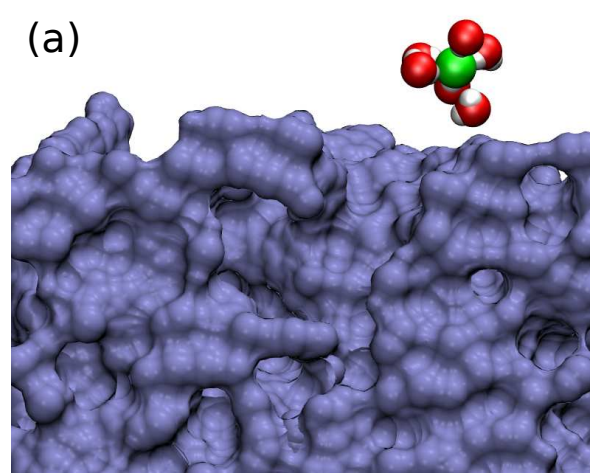

(c)

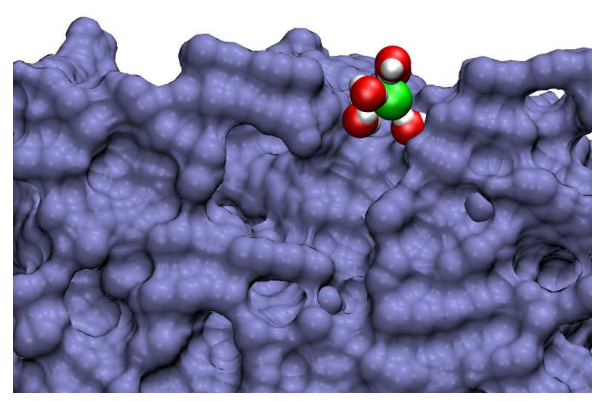

(e)

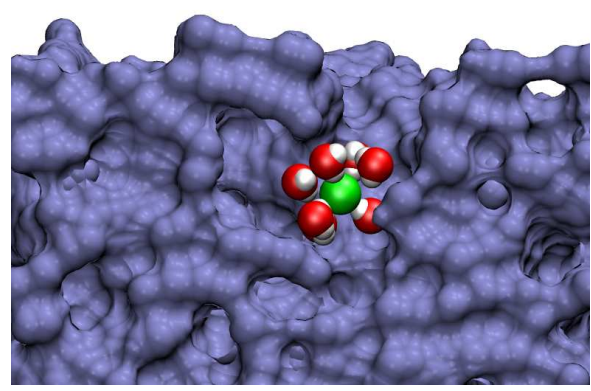

(b)

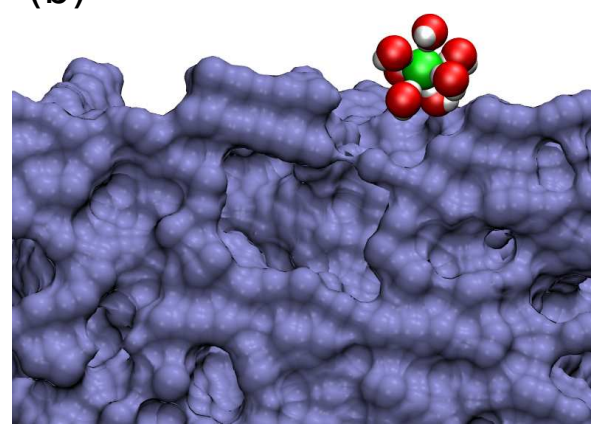

(d)

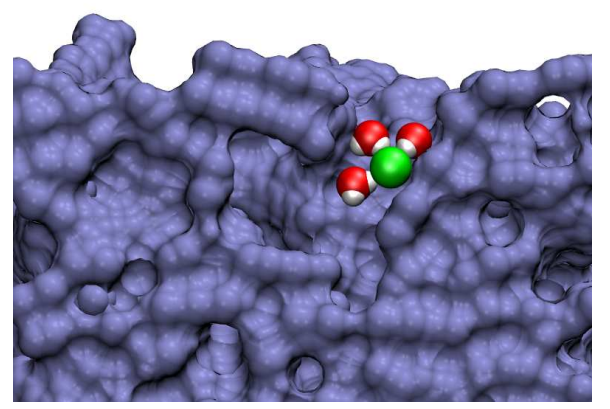

(f)

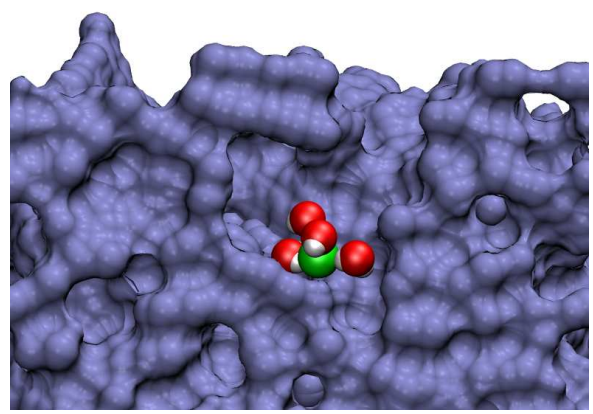

Figure 14: Snapshots taken from a sequence of umbrella sampling runs with a $\mathrm{Cl}^{-}$ion crossing the membrane-solution interface. The system is viewed looking down the $\mathrm{y}$-axis with the interface normal running vertically. The position of the chloride ion with respect to the membrane interface is (a) $8.4 \AA$, (b) $4.4 \AA$, (c) $-0.81 \AA$, (d) $-5.6 \AA$, (e) $-9.2 \AA$ and (f) $-12.4 \AA$, with negative position indicating that the ion is within the membrane. The water molecules, with the exception of those in the first solvation shell of the chloride ion, have been removed for clarity, the membrane has been coloured blue, the chloride ion green, the water oxygens and the water hydrogens red and white, respectively. The snapshots illustrate the type of pathway taken by both ions when diffusing across the membranesolution interface, with water molecules and polymer groups exchanging with each other in the solvation shell of the ion. 


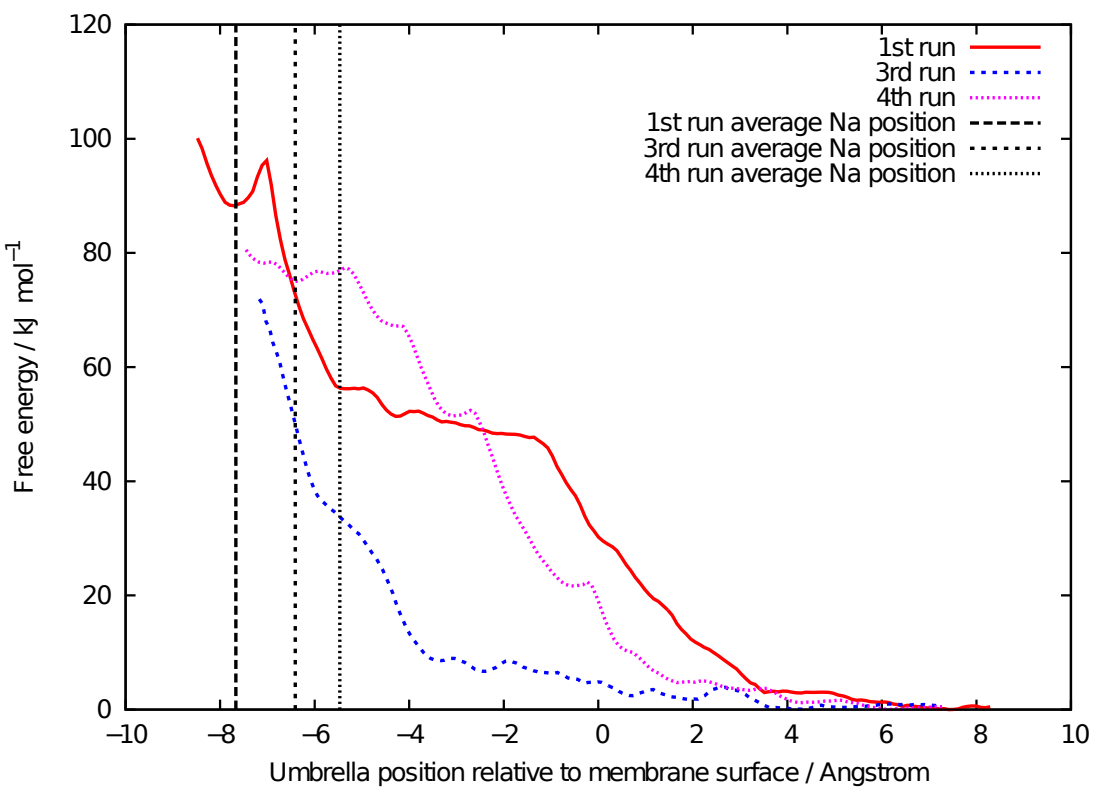

Figure 15: Free energy barrier for a $\mathrm{Cl}^{-}$ion to enter the FT30 membrane after a $\mathrm{Na}^{+}$ion has already entered the membrane, determined using umbrella sampling methods. The vertical lines show the average positions of the sodium ions throughout the three different umbrella sampling runs. 